\title{
The balloon-borne large-aperture submillimeter telescope for polarimetry-BLASTPol: performance and results from the 2010 Antarctic flight
}

Enzo Pascale ${ }^{a}$, Peter A. R. Ade ${ }^{a}$, Francesco E. Angilè ${ }^{b}$, Steven J. Benton ${ }^{c}$, Mark J. Devlin $^{b}$, Brad Dober $^{b}$, Laura M. Fissel ${ }^{c}$, Yasuo Fukui ${ }^{d}$, Natalie N. Gandilo ${ }^{c}$, Joshua O. Gundersen ${ }^{e}$,

Peter C. Hargrave ${ }^{a}$, Jeffrey Klein ${ }^{b}$, Andrei L. Korotkov ${ }^{f}$, Tristan G. Matthews ${ }^{g}$, Lorenzo Moncelsi $^{h, a}$, Tony K. Mroczkowski ${ }^{h}$, C. Barth Netterfield ${ }^{a, i}$, Giles Novak ${ }^{g}$, David Nutter $^{a}$, Luca Olmi ${ }^{j, k}$, Frédérick Poidevin ${ }^{l}$, Giorgio Savini ${ }^{l}$, Douglas Scott $^{m}$, Jamil A. Shariff ${ }^{b}$, Juan Diego Soler ${ }^{b}$, Nicholas E. Thomas ${ }^{e}$, Matthew D. P. Truch ${ }^{b}$, Carole E. Tucker ${ }^{a}$, Gregory S. Tucker ${ }^{f}$, Derek Ward-Thompson ${ }^{a}$

${ }^{a}$ School of Physics \& Astronomy, Cardiff University, 5 The Parade, Cardiff, CF24 3AA, UK;

${ }^{b}$ Department of Physics and Astronomy, University of Pennsylvania, 209 South 33rd Street,

Philadelphia, PA 19104, USA;

${ }^{c}$ Department of Astronomy \& Astrophysics, University of Toronto, 50 St. George Street,

Toronto, ON M5S 3H4, Canada;

${ }^{d}$ Department of Physics and Astrophysics, Nagoya University, Nagoya, Aichi 464-8601, Japan;

${ }^{e}$ Department of Physics, University of Miami, 1320 Campo Sano Drive, Coral Gables, FL 33146, USA;

fDepartment of Physics, Brown University, 182 Hope Street, Providence, RI 02912, USA;

${ }^{g}$ Department of Physics and Astronomy, Northwestern University, 2145 Sheridan Road,

Evanston, IL 60208-3112, USA;

${ }^{h}$ California Institute of Technology, 1200 E. California Blvd., Pasadena, CA 91125, USA;

${ }^{i}$ Department of Physics, University of Toronto, 60 St. George Street, Toronto, ON M5S 1A7, Canada;

jUniversity of Puerto Rico, Rio Piedras Campus, Physics Dept., Box 23343, UPR station, San Juan, Puerto Rico;

${ }^{k}$ Osservatorio Astrofisico di Arcetri, INAF, Largo E. Fermi 5, I-50125, Firenze, Italy.;

${ }^{l}$ Department of Physics and Astronomy, University College London, Gower Street, London, WC1E 6BT, UK;

${ }^{m}$ Department of Physics \& Astronomy, University of British Columbia, 6224 Agricultural Road, Vancouver, BC V6T 1Z1, Canada;

\begin{abstract}
The Balloon-borne Large Aperture Submillimeter Telescope for Polarimetry (BLASTPol) is a suborbital mapping experiment designed to study the role played by magnetic fields in the star formation process. BLASTPol uses a total power instrument and an achromatic half-wave plate to modulate the polarization signal. During its first flight from Antarctica in December 2010, BLASTPol made degree scale maps of linearly polarized dust emission from molecular clouds in three wavebands centered at 250,350, and $500 \mu \mathrm{m}$. This unprecedented dataset in terms of sky coverage, with sub-arcminute resolution, allows BLASTPol to trace magnetic fields in star-forming regions at scales ranging from cores to entire molecular cloud complexes. A second long-duration flight is scheduled for December 2012.
\end{abstract}

Further author information: (Send correspondence to E. Pascale)

E. pascale: E-mail: enzo.pascale@astro.cf.ac.uk, Telephone: +44 (0)29 20875031

Ground-based and Airborne Telescopes IV, edited by Larry M. Stepp, Roberto Gilmozzi, Helen J. Hall, Proc. of SPIE Vol. 8444, 844415 - (c) 2012 SPIE · CCC code: 0277-786/12/\$18 - doi: 10.1117/12.927211 
Keywords: submillimeter — stars: formation — instrumentation: miscellaneous — balloons — polarization dust emission

\section{INTRODUCTION}

The Balloon-borne Large Aperture Submillimeter Telescope for Polarimetry, BLASTPol, is a stratospheric $1.8 \mathrm{~m}$ diameter telescope which maps linearly polarized submillimeter emission with bolometric detectors operating in three $30 \%$ wide bands centered at 250,350, and $500 \mu \mathrm{m}$. BLASTPol's diffraction-limited optics were designed to provide a resolution of $30^{\prime \prime}, 42^{\prime \prime}$, and $60^{\prime \prime}$ at the three wavebands, respectively. The detectors and cold optics are adapted from those used by the SPIRE instrument on Herschel. ${ }^{11}$

The BLASTPol instrument ${ }^{9}$ is a rebuilt and enhanced version of the BLAST telescope, ${ }^{25}$ which was flown successfully in two long-duration balloon (LDB) campaigns ${ }^{17,29}$ and has left a legacy of important scientific results, many of which are still relevant in the Herschel era. . $^{18,21,26,27,31,34}$ BLAST was originally designed to conduct confusion-limited and wide-area extragalactic and Galactic surveys at submillimeter wavelengths from a LDB platform. These wavelengths are impossible or very difficult to observe from even the best ground-based telescope sites, but are accessible at stratospheric altitudes.

With the addition of a polarizing grid in front of each of the 266 feed horns at 250, 350 and $500 \mu \mathrm{m}$ and a stepped achromatic Half Wave Plate (AHWP), BLAST has been transformed into BLASTPol - an instrument designed to measure polarized dust emission from star forming regions. By mapping polarization from dust grains aligned with respect to their local magnetic field, the field orientation (projected on the sky) can be traced. The magnetic field strength can also be estimated indirectly using the polarization angular dispersion. ${ }^{4}$

During the first flight of BLASTPol in December 2010 from McMurdo, Antarctica, we made sensitive degreescale maps of several nearby molecular clouds. While the angular resolution $\left(<1.5^{\prime}\right)$ was worse than planned due to a blocking filter that was melted by the Sun on ascent, our preliminary polarization maps indicate coherent polarization across our target clouds at the few percent level. We show in Sec. 6 that our measurements on the Carina Nebula agree well with measurements from a much smaller and coarser resolution map made by a ground-based polarimeter. Similar maps of the Vela C and Lupus molecular clouds show that the flux is polarized at a few percent level and is detected with high significance.

This BLASTPol dataset will be used to investigate what role magnetic fields play in the star formation process, an important outstanding question in our understanding of how stars form. BLASTPol maps of magnetic fields across entire Giant Molecular Clouds (GMCs), have sufficient resolution to probe fields in dense filamentary sub-structures and molecular cores. The experiment provides a crucial bridge between the large area but coarse resolution polarimetry provided by experiments such as Planck and the small area but high resolution of the ALMA telescope.

A second flight is scheduled to occur in December 2012 from Antarctica to improve on the area, depth and angular resolution obtained during the 2010 flight.

\section{PROBING THE ROLES OF MAGNETIC FIELDS IN STAR FORMATION}

One of the key goals of modern astrophysics is to understand the details of star formation: how their masses are determined and what are the dominant physical processes that regulate the overall rate of star formation in the Galaxy. Significant progress has been made on these questions in recent years. For example, observations of dust emission and extinction ${ }^{22}$ show that the overall distribution of core masses mimics the distribution of stellar masses. Recent Herschel observations have shown that molecular clouds present an ubiquitous filamentary structure, in which long thin filaments form first, and then fragment into pre-stellar cores. ${ }^{1,14}$ However, fundamental questions regarding molecular cloud structure and star formation are still being debated. ${ }^{19}$ For example, some investigators argue that molecular clouds, as well as cores, clumps, and filaments inside the clouds, are dynamic structures, whose lifetimes are approximately equal to their turbulent crossing times. ${ }^{30}$ Others favor longer lifetimes, of order several crossing times. ${ }^{2,10,21}$ If clouds and their sub-structures do indeed live longer than a crossing time, they require support against gravity; this support could be provided by magnetic fields, which in many numerical simulations dramatically affect both the star formation efficiency and the life-time of 


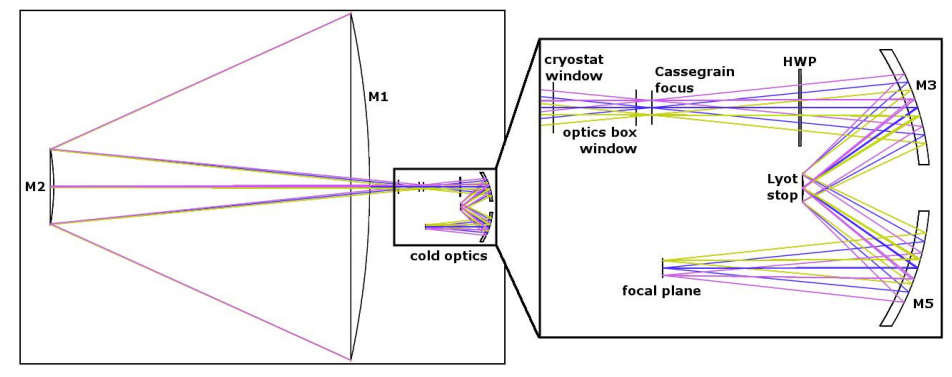

Figure 1. Schematic of the optical layout for the BLASTPol telescope and receiver is shown on the left, with the $1.5 \mathrm{~K}$ optics, located within the cryostat, shown in an expanded view on the right. The image of the sky formed at the input aperture is re-imaged onto the bolometer detector arrays at the focal plane. The M4 mirror serves as a Lyot stop, which defines the illumination of the primary mirror for each element of the bolometer detector arrays. The three wavelength bands are separated by a pair of dichroic beam-splitters (not shown here). The sapphire half-wave plate is also shown, mounted $19.174 \mathrm{~cm}$ from the focus of the telescope.

molecular clouds. ${ }^{12,16}$ However, observationally, the strength and morphology of magnetic fields in molecular clouds have been poorly constrained. Zeeman splitting detections are limited to the brightest Galactic sources ${ }^{6,8}$ and optical polarimetry is not possible in these regions of high extinction. The best method for probing these fields is far-IR and submillimeter polarimetry, ${ }^{13,32,33}$ where the radiation from asymmetric dust grains, aligned by the local magnetic field, is detected in polarization. BLASTPol is the first submillimeter polarimeter with both sufficient mapping speed to trace fields across entire clouds and sub-arcminute spatial resolution, to trace the fields onto the scale of dense cores. This provides a critical link between the Planck all-sky polarization maps (with $5^{\prime}$ resolution) and the planned ALMA polarization measurements at ultra-high resolution of small individual sources (though with only a $20^{\prime \prime}$ field of view). BLASTPol data will allow the first comprehensive detailed comparisons between observed molecular cloud magnetic fields, and models derived from numerical simulations. ${ }^{23}$ Recent observations show that the extended sub-millimeter emission from molecular clouds is indeed polarized, ${ }^{15,32,33}$ and BLASTPol data is in good agreement with some of these early results (see Sec. 6).

BLASTPol observations target the following three key questions in star formation and are discussed in Ref. 9: i) Is core morphology and evolution determined by large-scale magnetic fields? ii) Does filamentary structure have a magnetic origin? iii) What is the field strength, and how does it vary from cloud to cloud?

\section{INSTRUMENT}

The BLASTPol instrument is a modification of the BLAST telescope that adds linear polarization capabilities. A detailed description is given in Ref. 9,17,25. The main features of the optical system are summarized in Fig. 1. The Ritchey-Chrétien telescope has an aluminum primary mirror with a diameter of $1.8 \mathrm{~m}$. The radiation collected is re-imaged by a series of cold $(\sim 1.5 \mathrm{~K})$ reflecting optical elements arranged into an ideal Offner relay inside a long duration cryostat. This cryostat uses liquid helium and nitrogen and has a hold time of more than 10 days. The telescope's secondary mirror is actuated, so the system can be refocused in-flight. The light is split into three $30 \%$ wide submillimetric bands respectively centered at 250, 350 and $500 \mu \mathrm{m}$. The BLASTPol focal plane consists of arrays of 149,88 , and 43 detectors at 250,350, and $500 \mu \mathrm{m}$, respectively. The arrays are cooled to a temperature of $270 \mathrm{mK}$. Each array element is a silicon nitride micromesh "spiderweb" bolometer, ${ }^{3}$ coupled to the front optics by a smooth-walled conical feed-horn. ${ }^{5}$ Linear polarimetry is achieved by adding a polarizing grid at the mouth of each feed-horn, and a stepped achromatic half-wave plate (AHWP) to modulate the polarization.

Each target is observed in a slow raster-scan mode. Slow scanning is preferable to a mechanical chopper for mapping large regions of the sky. The telescope is scanned in azimuth at a constant velocity of $\sim 0.05^{\circ} / \mathrm{s}$. At the end of each azimuthal scan, the elevation is stepped by $1 / 3$ of the array's $7^{\prime}$ field of view (FOV) in elevation (the array FOV is $14^{\prime} \times 7^{\prime}$ ). 

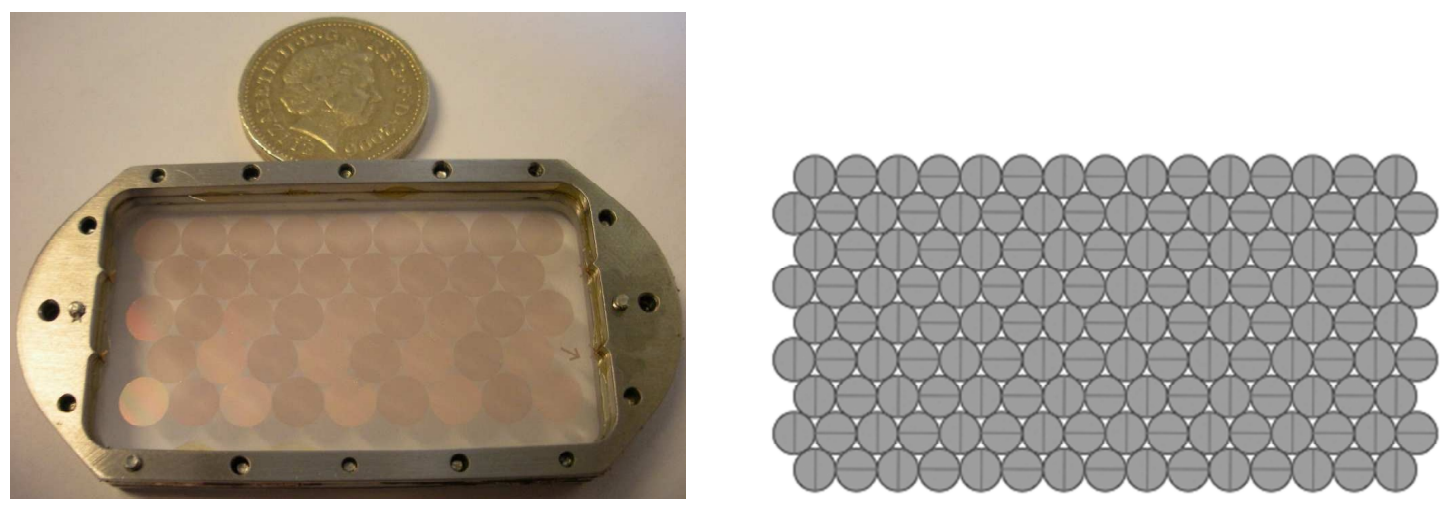

Figure 2. Left: Photolithographed polarization grid for the $500 \mu \mathrm{m}$ feed-horn array. Right : Scheme of the $90^{\circ}$-alternated grid pattern along the scan direction.

\section{POLARIMETRY}

A photo-lithographed polarizing grid (Fig. 2) is mounted in front of the feed-horn arrays for each bolometer detector array. The grids are patterned to alternate the polarization angle sampled by $90^{\circ}$ from horn-to-horn and thus bolometer-to-bolometer along the scan direction. This arrangement has proved effective in rejecting $1 / f$ noise correlated among detectors in an array (array common modes). BLASTPol scans so that a source on the sky passes along a row of detectors, and thus the time required to measure one Stokes parameter $(Q$ or $U)$ is just equal to the separation between bolometers divided by the scan speed. For the $250 \mu \mathrm{m}$ detector array where the bolometers are separated by $45^{\prime \prime}$, and assuming a typical scan speed of $0.05^{\circ} / \mathrm{s}$, this time is $0.25 \mathrm{~s}$. This timescale is short compared to the characteristic low frequency $(1 / f)$ noise knee for the detectors at $0.035 \mathrm{mHz} .^{25}$ Each grid is illuminated by the almost-Gaussian field launched by each feed-horn. In this way, the illumination is tapered at the edge of each grid such that cross-polar response which could arise from partially illuminating a $90^{\circ}$ rotated adjacent grid is negligible. The efficiency of the grid is $97 \%$ or better, while the cross polarization is estimated to be always less than $0.07 \% .^{20}$

The use of a stepped AHWP allows modulation of the Stokes parameters $Q$ and $U$ such that each detector measures $I, Q$, and $U$ multiple times in each sky direction. A total of four AHWP position angles are used (at $0,22.5,45$, and $67.5^{\circ}$ ), stepped at the end of the telescope's raster-scan on a given target. This mitigates the effect of unbalanced gains between adjacent detectors which would result in a large bias on the estimated $Q$ and $U$ if only detector differences are used to estimate the Stokes parameters.

The BLASTPol AHWP is $10 \mathrm{~cm}$ in diameter and is constructed from 5 plates of sapphire, each $500 \mu \mathrm{m}$ in thickness. Each plate is cut so that the extraordinary axis is parallel to the surfaces of the plates. The plates are glued together with a $6 \mu \mathrm{m}$ layer of polyethylene following a modified Pancharatnam ${ }^{24}$ design optimized for achromaticity and efficiency across the three BLASTPol bands. An anti-reflection coating, made from metal mesh filter technology ${ }^{35}$ is glued to each surface of the half-wave plate.

The AHWP is in the cryostat at a temperature of $4 \mathrm{~K}(1)$ and is mounted in the optical path between the main telescope and the re-imaging optics. Its performance is assessed using a comprehensive set of data consisting of spectra taken with a polarizing Fourier Transform Spectrometer. Measurements are taken with the HWP at temperatures of $300 \mathrm{~K}$ and $120 \mathrm{~K}$, and are extrapolated to predict the $4 \mathrm{~K}$ performance. Following the methodology described in Ref. 20 (see also Moncelsi et al., in prep.), the 9 elements of the AHWP Mueller matrix associated with linear polarization are experimentally measured, and are shown in Fig. 3 for a source with a flat spectral energy distribution (SED). The Mueller matrix is close to ideal, with the off-diagonal elements below $2 \%$ in all three bands. This means a low instrumental polarization (IP). The optical and polarization efficiencies are measured by the three diagonal elements of the matrix. At 350 and $500 \mu \mathrm{m}$ the optical efficiency is $\simeq 1$ and the polarization efficiency is larger than $95 \%$. At $250 \mu \mathrm{m}$ the in-band optical efficiency is $\simeq 0.9$ and the polarization efficiency is $\simeq 80 \%$. This reduced performance at the shortest wavelength band is expected and is due to the requirement for the exceptionally large spectral range of BLASTPol. Scientific considerations suggest 

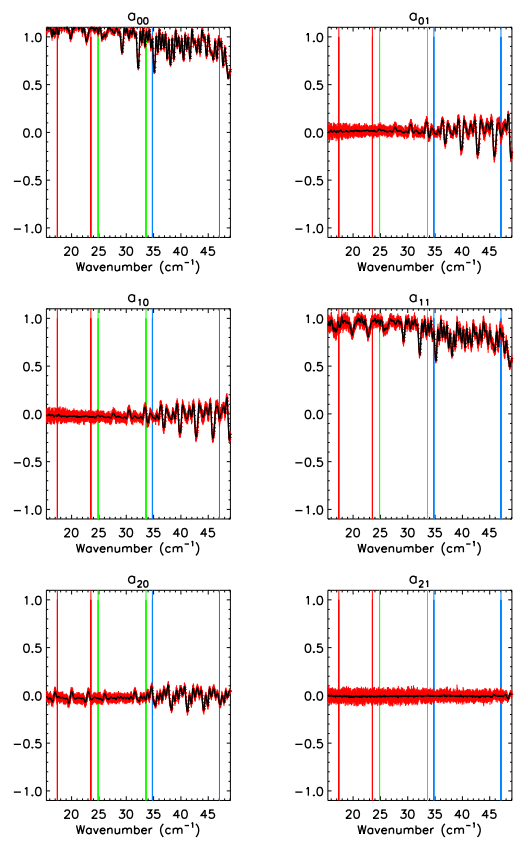
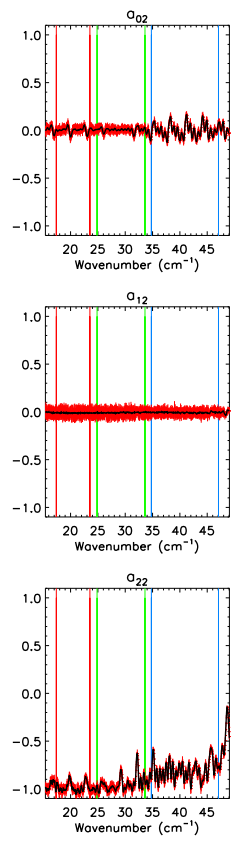

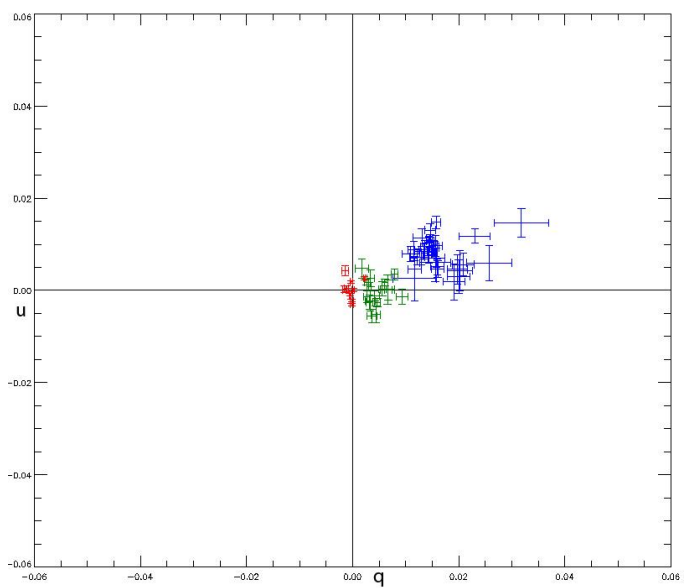

Figure 3. Left: Graphical representation of the 9-element Mueller matrix elements $a_{i j}$ of the $4 \mathrm{~K}$ cold BLASTPol AHWP as a function of wavenumber. ${ }^{20}$ A flat-spectrum source is assumed. The $10 \sigma$ error bars are shown in red. The three BLASTPol bands centered at 250, 350 and $500 \mu \mathrm{m}$ are shown by the vertical lines. Each detector is sensitive to the in-band integral of the matrix elements, weighted by the source SED. Right: The measured IP for the fully assembled receiver (AHWP, re-imaging optics, polarizing grids and feed-horn coupled detectors). The measured IP is less than $1 \%$ at 500 and $350 \mu \mathrm{m}$ (red and green data-points, respectively), and less than $2 \%$ at $250 \mu \mathrm{m}$ (blue data-points).

that a optimal performance at $500 \mu \mathrm{m}$ is preferred, and the system has been optimized for operations at this wavelength.

Measurements of IP were performed before flight for the integrated cold optical system comprising the AHWP, re-imaging optics, the polarization grids and the feed-coupled detectors. An unpolarized black body source is chopped in front of the receiver and the instrumentally induced polarization is estimated. The result is shown in Fig. 3 and confirms a very low IP in the three bands. A slightly higher IP is detected in the $250 \mu \mathrm{m}$ band, as predicted, but it is still well below $2 \%$. The on-axis telescope is not a significant source of IP and therefore an overall small IP is predicted for the whole instrument.

In-flight, the AHWP is operated in a stepped mode, rather than a continuously rotating mode. The rotator employs a pair of thin-section steel ball bearings housed in a stainless steel structure, and is driven via a gear train and a G10 fiberglass shaft leading to a stepper motor outside the cryostat. A ferrofluidic vacuum seal is used for the drive shaft. The angle sensing at liquid helium temperatures is accomplished by a potentiometer element making light contact with phosphor bronze leaf springs. The rotator and encoder are based on a design used successfully at the South Pole. ${ }^{28}$

\section{FLIGHTS}

The BLAST program has had three LDB flights. In 2005 BLAST was the first large payload to be launched from Kiruna, Sweden. The instrument was then refurbished with a new telescope and flown again in 2006 from McMurdo Antarctica. These first two flights produced a host of high-profile results including a measurement of the FIR background at 250,350, and $500 \mu \mathrm{m}^{7,18,26}$ and a map of the Vela Molecular Cloud ${ }^{21}$ showing that prestellar cores require a support mechanism to explain the observed long life-times. Magnetic support is one of the possible mechanisms which can provide such support and one of BLASTPol's main science drivers is to investigate this assumption. 


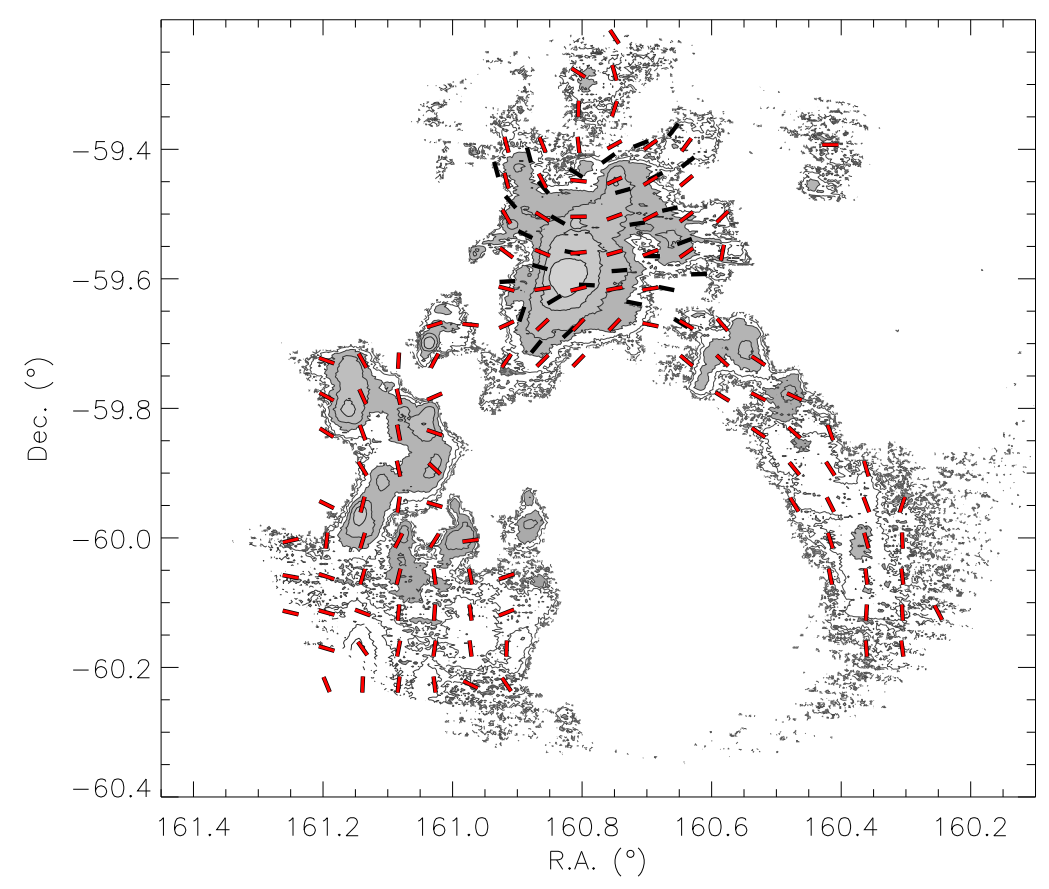

Figure 4. Preliminary results from the BLASTPol 2010 fight. The lines indicate the inferred magnetic field direction. Here we show a map made from 3 hours of BLASTPol data on the Carina Nebula. Total intensity contour levels measured at $500 \mu \mathrm{m}$ are shown in the background. The red pseudo-vectors are BLASTPol measurements. The black pseudo-vectors are measurements from the SPARO polarimeter at $450 \mu \mathrm{m}^{15}$ showing a good agreement between the two detections.

During the first fight of BLASTPol in 2010 we made sensitive degree-scale maps of several nearby molecular clouds. Our preliminary polarization maps indicate coherent polarization across our target clouds at the few percent level. The Galactic targets observed during this 9 day flight are listed in Table 1. *

Preliminary results from the data-reduction effort of the 2010 dataset indicate that our measurements on the Carina nebula agree well with measurements from a much smaller and coarser resolution map made by a ground- based polarimeter (Fig. 4). For the nearby Vela C molecular cloud there is good agreement between all three BLASTPol bands indicating that the flux is polarized at the $2 \%$ level. Similar results are emerging for the Lupus Molecular Cloud and other targets observed in 2010.

For the 2010 flight we used a conservative approach to target selection by limiting the number and map size for our science targets. The reasons for our caution were that we did not know the polarization level of our target clouds, and also the 2010 flight was our first opportunity to test the effectiveness of BLASTPol in reconstructing polarization fields. As the emission of our target molecular clouds appears to be polarized at the few percent level and BLASTPol has proved successful as a polarimeter, a second Antarctic flight has been scheduled for December 2012 where a larger dataset, in terms of both mapped area and total number of science targets, will be observed.

\section{DATA REDUCTION AND PRELIMINARY RESULTS}

The reduction of the bolometric timelines into polarization maps is done using the well-tested BLAST pipeline, modified to include polarization. The process is described in the References 25 and 27 and involves cleaning the timelines for cosmic-rays and other glitches by flagging these spurious events which are not projected into the map. Common modes arising from the telescope elevation motion are also decorrelated in the time domain. After subtracting the known IP, the clean timelines are then binned into the three Stokes maps of $I, Q$, and $U$, by inverting the polarized pixel-binning matrix which accumulates the polarization angles by the scan. ${ }^{20}$ This is

${ }^{*}$ See also http://blastexperiment.info. 
Table 1. BLASTPol 2010 Observed Targets

\begin{tabular}{|l|c|c|c|}
\hline Target & $\begin{array}{c}\text { Distance } \\
(\mathrm{pc})\end{array}$ & $\begin{array}{c}\text { Obs. Time } \\
\text { (hours) }\end{array}$ & No. of B-vectors \\
\hline Vela Molecular Ridge & $\sim 700$ & 64 & $\sim 1,000$ \\
Lupus Cloud Complex & $\sim 200$ & 62 & $\sim 200$ \\
Puppis Cloud Complex & $\sim 1,000$ & 22 & $\sim 50$ \\
IRDC filaments & $\sim 2,000-4,000$ & 8 & $\sim 100$ \\
cool GMCs & $\sim 3,000-5,000$ & 18 & $\sim 200$ \\
Carina Nebula & $\sim 3,000$ & 3 & $\sim 100$ \\
\hline
\end{tabular}

equivalent to a naive binning of the data. Stokes parameter maps are then used to reconstruct the polarization field in the form of total polarization fractions and polarization angles. The direction of the magnetic field component projected on the plane of the sky is then inferred by rotating the polarization pseudo-vectors by $90^{\circ}$.

This is a reliable data-reduction approach, as it is shown in Fig. 4 where the BLASTPol reconstructed magnetic field is in very good agreement with previous measurements at a similar wavelength, despite reduced performance in angular resolution arising from a problem with the blocking filter. Similar results are being obtained on the other main science targets observed during the 2010 flight. Currently, we are investigating the reliability of all reconstructed pseudo-vectors using an aggressive program of simulations and data jackknife tests. This work in progress has shown that polarization is detected at the few percent level on the other science targets for which independent submillimetric polarization data is not available. We plan to publish these findings in the current year.

\section{ACKNOWLEDGMENTS}

The BLAST collaboration acknowledges the support of NASA through grant numbers NAG5-12785, NAG513301 and NNGO-6GI11G, the Canadian Space Agency (CSA), the Leverhulme Trust through the Research Project Grant F/00 407/BN, Canada's Natural Sciences and Engineering Research Council (NSERC), the Canada Foundation for Innovation, the Ontario Innovation Trust, the Puerto Rico Space Grant Consortium, the Fondo Istitucional para la Investigacion of the University of Puerto Rico, and the National Science Foundation Office of Polar Programs; C. B. Netterfield also acknowledges support from the Canadian Institute for Advanced Research. We would also like to thank the Columbia Scientific Balloon Facility (CSBF) staff for their outstanding work.

\section{REFERENCES}

[1] P. André, A. Men'shchikov, S. Bontemps, V. Könyves, F. Motte, N. Schneider, P. Didelon, V. Minier, P. Saraceno, D. Ward-Thompson, J. di Francesco, G. White, S. Molinari, L. Testi, A. Abergel, M. Griffin, T. Henning, P. Royer, B. Merín, R. Vavrek, M. Attard, D. Arzoumanian, C. D. Wilson, P. Ade, H. Aussel, J.-P. Baluteau, M. Benedettini, J.-P. Bernard, J. A. D. L. Blommaert, L. Cambrésy, P. Cox, A. di Giorgio, P. Hargrave, M. Hennemann, M. Huang, J. Kirk, O. Krause, R. Launhardt, S. Leeks, J. Le Pennec, J. Z. Li, P. G. Martin, A. Maury, G. Olofsson, A. Omont, N. Peretto, S. Pezzuto, T. Prusti, H. Roussel, D. Russeil, M. Sauvage, B. Sibthorpe, A. Sicilia-Aguilar, L. Spinoglio, C. Waelkens, A. Woodcraft, and A. Zavagno, From filamentary clouds to prestellar cores to the stellar IMF: Initial highlights from the Herschel Gould Belt Survey, A\&A518 (2010), L102.

[2] L. Blitz, Y. Fukui, A. Kawamura, A. Leroy, N. Mizuno, and E. Rosolowsky, Giant Molecular Clouds in Local Group Galaxies, Protostars and Planets V (B. Reipurth, D. Jewitt, and K. Keil, eds.), 2007, pp. 81-96.

[3] J. J. Bock, J. Glenn, S. M. Grannan, K. D. Irwin, A. E. Lange, H. G. Leduc, and A. D. Turner, Silicon nitride micromesh bolometer arrays for SPIRE, Society of Photo-Optical Instrumentation Engineers (SPIE) Conference Series (T. G. Phillips, ed.), Society of Photo-Optical Instrumentation Engineers (SPIE) Conference Series, vol. 3357, July 1998, pp. 297-304.

[4] S. Chandrasekhar and E. Fermi, Magnetic Fields in Spiral Arm, ApJ118 (1953), 113. 
[5] G. Chattopadhyay, J. Glenn, J. J. Bock, B. K. Rownd, M. Caldwell, and M. J. Griffin 2003, Feed horn coupled bolometer arrays for spire - design, simulations, and measurements, IEEE Trans. Micro. T. Tech (2003).

[6] R. M. Crutcher, B. Wandelt, C. Heiles, E. Falgarone, and T. H. Troland, Magnetic Fields in Interstellar Clouds from Zeeman Observations: Inference of Total Field Strengths by Bayesian Analysis, ApJ725 (2010), 466-479.

[7] M. J. Devlin, P. A. R. Ade, I. Aretxaga, J. J. Bock, E. L. Chapin, M. Griffin, J. O. Gundersen, M. Halpern, P. C. Hargrave, D. H. Hughes, J. Klein, G. Marsden, P. G. Martin, P. Mauskopf, L. Moncelsi, C. B. Netterfield, H. Ngo, L. Olmi, E. Pascale, G. Patanchon, M. Rex, D. Scott, C. Semisch, N. Thomas, M. D. P. Truch, C. Tucker, G. S. Tucker, M. P. Viero, and D. V. Wiebe, Over half of the far-infrared background light comes from galaxies at $z \geq 1$.2, Nature 458 (2009), 737-739.

[8] E. Falgarone, T. H. Troland, R. M. Crutcher, and G. Paubert, CN Zeeman measurements in star formation regions, A\&BA487 (2008), 247-252.

[9] L. M. Fissel, P. A. R. Ade, F. E. Angilè, S. J. Benton, E. L. Chapin, M. J. Devlin, N. N. Gandilo, J. O. Gundersen, P. C. Hargrave, D. H. Hughes, J. Klein, A. L. Korotkov, G. Marsden, T. G. Matthews, L. Moncelsi, T. K. Mroczkowski, C. B. Netterfield, G. Novak, L. Olmi, E. Pascale, G. Savini, D. Scott, J. A. Shariff, J. D. Soler, N. E. Thomas, M. D. P. Truch, C. E. Tucker, G. S. Tucker, D. Ward-Thompson, and D. V. Wiebe, The balloon-borne large-aperture submillimeter telescope for polarimetry: BLAST-Pol, Society of Photo-Optical Instrumentation Engineers (SPIE) Conference Series, Society of Photo-Optical Instrumentation Engineers (SPIE) Conference Series, vol. 7741, July 2010.

[10] P. F. Goldsmith, M. Heyer, G. Narayanan, R. Snell, D. Li, and C. Brunt, Large-Scale Structure of the Molecular Gas in Taurus Revealed by High Linear Dynamic Range Spectral Line Mapping, ArXiv e-prints 802 (2008).

[11] M. J. Griffin, B. M. Swinyard, and L. G. Vigroux, SPIRE - Herschel's Submillimetre Camera and Spectrometer, IR Space Telescopes and Instruments. Edited by John C. Mather . Proceedings of the SPIE, vol. 4850, March 2003, pp. 686-697.

[12] P. Hennebelle, B. Commerçon, M. Joos, R. S. Klessen, M. Krumholz, J. C. Tan, and R. Teyssier, Collapse, outflows and fragmentation of massive, turbulent and magnetized prestellar barotropic cores, A\&A528 (2011), A72.

[13] R. H. Hildebrand, J. A. Davidson, J. L. Dotson, C. D. Dowell, G. Novak, and J. E. Vaillancourt, A Primer on Far-Infrared Polarimetry, PASP112 (2000), 1215-1235.

[14] T. Hill, F. Motte, P. Didelon, S. Bontemps, V. Minier, M. Hennemann, N. Schneider, P. André, A. Men'shchikov, L. D. Anderson, D. Arzoumanian, J.-P. Bernard, J. di Francesco, D. Elia, T. Giannini, M. J. Griffin, V. Könyves, J. Kirk, A. P. Marston, P. G. Martin, S. Molinari, Q. Nguyen Luong, N. Peretto, S. Pezzuto, H. Roussel, M. Sauvage, T. Sousbie, L. Testi, D. Ward-Thompson, G. J. White, C. D. Wilson, and A. Zavagno, Filaments and ridges in Vela C revealed by Herschel: from low-mass to high-mass star-forming sites, Aछ̈A533 (2011), A94.

[15] H. Li, G. S. Griffin, M. Krejny, G. Novak, R. F. Loewenstein, M. G. Newcomb, P. G. Calisse, and D. T. Chuss, Results of SPARO 2003: Mapping Magnetic Fields in Giant Molecular Clouds, ApJ648 (2006), 340-354.

[16] Z.-Y. Li, P. Wang, T. Abel, and F. Nakamura, Lowering the Characteristic Mass of Cluster Stars by Magnetic Fields and Outflow Feedback, ApJ720 (2010), L26-L30.

[17] G. Marsden, P. A. R. Ade, S. Benton, J. J. Bock, E. L. Chapin, J. Chung, M. J. Devlin, S. Dicker, L. Fissel, M. Griffin, J. O. Gundersen, M. Halpern, P. C. Hargrave, D. H. Hughes, J. Klein, A. Korotkov, C. J. MacTavish, P. G. Martin, T. G. Martin, T. G. Matthews, P. Mauskopf, L. Moncelsi, C. B. Netterfield, G. Novak, E. Pascale, L. Olmi, G. Patanchon, M. Rex, G. Savini, D. Scott, C. Semisch, N. Thomas, M. D. P. Truch, C. Tucker, G. S. Tucker, M. P. Viero, D. Ward-Thompson, and D. V. Wiebe, The Balloon-borne LargeAperture Submillimeter Telescope for polarization: BLAST-pol, Society of Photo-Optical Instrumentation Engineers (SPIE) Conference Series, Society of Photo-Optical Instrumentation Engineers (SPIE) Conference Series, vol. 7020, August 2008. 
[18] G. Marsden, P. A. R. Ade, J. J. Bock, E. L. Chapin, M. J. Devlin, S. R. Dicker, M. Griffin, J. O. Gundersen, M. Halpern, P. C. Hargrave, D. H. Hughes, J. Klein, P. Mauskopf, B. Magnelli, L. Moncelsi, C. B. Netterfield, H. Ngo, L. Olmi, E. Pascale, G. Patanchon, M. Rex, D. Scott, C. Semisch, N. Thomas, M. D. P. Truch, C. Tucker, G. S. Tucker, M. P. Viero, and D. V. Wiebe, BLAST: Resolving the Cosmic Submillimeter Background, ApJ707 (2009), 1729-1739.

[19] C. F. McKee and E. C. Ostriker, Theory of Star Formation, ARA $3 A 45$ (2007), 565-687.

[20] L. Moncelsi, BLAST: studying cosmic and Galactic star formation from a stratospheric balloon, Ph.D. thesis, Cardiff University, UK, URL: http://orca.cf.ac.uk/13144/, 2011.

[21] C. B. Netterfield, P. A. R. Ade, J. J. Bock, E. L. Chapin, M. J. Devlin, M. Griffin, J. O. Gundersen, M. Halpern, P. C. Hargrave, D. H. Hughes, J. Klein, G. Marsden, P. G. Martin, P. Mauskopf, L. Olmi, E. Pascale, G. Patanchon, M. Rex, A. Roy, D. Scott, C. Semisch, N. Thomas, M. D. P. Truch, C. Tucker, G. S. Tucker, M. P. Viero, and D. V. Wiebe, BLAST: The Mass Function, Lifetimes, and Properties of Intermediate Mass Cores from a $50 \mathrm{deg}^{2}$ Submillimeter Galactic Survey in Vela (ell 265 ), ApJ707 (2009), 1824-1835.

[22] D. Nutter and D. Ward-Thompson, A SCUBA survey of Orion - the low-mass end of the core mass function, MNRAS374 (2007), 1413-1420.

[23] C. Ostriker, J. M. Stone, and C. F. Gammie, Density, Velocity, and Magnetic Field Structure in Turbulent Molecular Cloud Models, ApJ546 (2001), 980-1005.

[24] S. Pancharatnam, Achromatic Combinations of Birefringent Plates: Part II. An Achromatic Quarter-Wave Plate, Memoirs of the Raman Research Institute Bangalore 71 (1955), 137-144.

[25] E. Pascale, P. A. R. Ade, J. J. Bock, E. L. Chapin, J. Chung, M. J. Devlin, S. Dicker, M. Griffin, J. O. Gundersen, M. Halpern, P. C. Hargrave, D. H. Hughes, J. Klein, C. J. MacTavish, G. Marsden, P. G. Martin, T. G. Martin, P. Mauskopf, C. B. Netterfield, L. Olmi, G. Patanchon, M. Rex, D. Scott, C. Semisch, N. Thomas, M. D. P. Truch, C. Tucker, G. S. Tucker, M. P. Viero, and D. V. Wiebe, The Balloon-borne Large Aperture Submillimeter Telescope: BLAST, ApJ681 (2008), 400-414.

[26] E. Pascale, P. A. R. Ade, J. J. Bock, E. L. Chapin, M. J. Devlin, S. Dye, S. A. Eales, M. Griffin, J. O. Gundersen, M. Halpern, P. C. Hargrave, D. H. Hughes, J. Klein, G. Marsden, P. Mauskopf, L. Moncelsi, H. Ngo, C. B. Netterfield, L. Olmi, G. Patanchon, M. Rex, D. Scott, C. Semisch, N. Thomas, M. D. P. Truch, C. Tucker, G. S. Tucker, M. P. Viero, and D. V. Wiebe, BLAST: A Far-Infrared Measurement of the History of Star Formation, ApJ707 (2009), 1740-1749.

[27] G. Patanchon, P. A. R. Ade, J. J. Bock, E. L. Chapin, M. J. Devlin, S. R. Dicker, M. Griffin, J. O. Gundersen, M. Halpern, P. C. Hargrave, D. H. Hughes, J. Klein, G. Marsden, P. Mauskopf, L. Moncelsi, C. B. Netterfield, L. Olmi, E. Pascale, M. Rex, D. Scott, C. Semisch, N. Thomas, M. D. P. Truch, C. Tucker, G. S. Tucker, M. P. Viero, and D. V. Wiebe, Submillimeter Number Counts from Statistical Analysis of BLAST Maps, ApJ 707 (2009), 1750-1765.

[28] T. Renbarger, D. T. Chuss, J. L. Dotson, G. S. Griffin, J. L. Hanna, R. F. Loewenstein, P. S. Malhotra, J. L. Marshall, G. Novak, and R. J. Pernic, Early Results from SPARO: Instrument Characterization and Polarimetry of NGC 6334, PASP116 (2004), 415-424.

[29] M. D. P. Truch, P. A. R. Ade, J. J. Bock, E. L. Chapin, M. J. Devlin, S. R. Dicker, M. Griffin, J. O. Gundersen, M. Halpern, P. C. Hargrave, D. H. Hughes, J. Klein, G. Marsden, P. G. Martin, P. Mauskopf, L. Moncelsi, C. Barth Netterfield, L. Olmi, E. Pascale, G. Patanchon, M. Rex, D. Scott, C. Semisch, N. E. Thomas, C. Tucker, G. S. Tucker, M. P. Viero, and D. V. Wiebe, The Balloon-borne Large Aperture Submillimeter Telescope (BLAST) 2006: Calibration and Flight Performance, ApJ707 (2009), 1723-1728.

[30] E. Vázquez-Semadeni, D. Ryu, T. Passot, R. F. González, and A. Gazol, Molecular Cloud Evolution. I. Molecular Cloud and Thin Cold Neutral Medium Sheet Formation, ApJ643 (2006), 245-259.

[31] M. P. Viero, P. A. R. Ade, J. J. Bock, E. L. Chapin, M. J. Devlin, M. Griffin, J. O. Gundersen, M. Halpern, P. C. Hargrave, D. H. Hughes, J. Klein, C. J. MacTavish, G. Marsden, P. G. Martin, P. Mauskopf, L. Moncelsi, M. Negrello, C. B. Netterfield, L. Olmi, E. Pascale, G. Patanchon, M. Rex, D. Scott, C. Semisch, N. Thomas, M. D. P. Truch, C. Tucker, G. S. Tucker, and D. V. Wiebe, BLAST: Correlations in the Cosmic Far-Infrared Background at 250, 350, and $500 \mu \mathrm{m}$ Reveal Clustering of Star-forming Galaxies, ApJ707 (2009), 1766-1778. 
[32] D. Ward-Thompson, J. M. Kirk, R. M. Crutcher, J. S. Greaves, W. S. Holland, and P. André, First Observations of the Magnetic Field Geometry in Prestellar Cores, ApJ537 (2000), L135-L138.

[33] D. Ward-Thompson, A. K. Sen, J. M. Kirk, and D. Nutter, Optical and submillimetre observations of Bok globules - tracing the magnetic field from low to high density, MNRAS398 (2009), 394-400.

[34] D. V. Wiebe, P. A. R. Ade, J. J. Bock, E. L. Chapin, M. J. Devlin, S. Dicker, M. Griffin, J. O. Gundersen, M. Halpern, P. C. Hargrave, D. H. Hughes, J. Klein, G. Marsden, P. G. Martin, P. Mauskopf, C. B. Netterfield, L. Olmi, E. Pascale, G. Patanchon, M. Rex, D. Scott, C. Semisch, N. Thomas, M. D. P. Truch, C. Tucker, G. S. Tucker, and M. P. Viero, BLAST Observations of Resolved Galaxies: Temperature Profiles and the Effect of Active Galactic Nuclei on FIR to Submillimeter Emission, ApJ707 (2009), 1809-1823.

[35] J. Zhang, P. A. R. Ade, P. Mauskopf, L. Moncelsi, G. Savini, and N. Whitehouse, New artificial dielectric metamaterial and its application as a terahertz antireflection coating, Appl. Opt.48 (2009), 6635-+. 